\title{
ESTIMATION OF THE MEAN VALUE IN A MODEL OF MIXTURES WITH VARYING CONCENTRATIONS
}

UDC 519.21

\author{
A. SHCHERBINA
}

\begin{abstract}
We consider a model for observations sampled from a two-component mixture. A certain numerical characteristic corresponds to every object. The distribution of the characteristic is unknown. The observations are obtained by sampling objects belonging to several groups. This procedure results in dependences among characteristics of objects, which constitute the difference between this setting and the classical one. The total amount of objects of the first and second class in each group is known, while the classes of objects are unknown. We consider the problem of estimation of mean values for characteristics of objects in each class. Two different settings are considered and expressions for the mean square errors are obtained for each of the settings. We show that the corresponding estimators are consistent and asymptotically normal.
\end{abstract}

\section{INTRODUCTION}

Several natural problems of the analysis of mixtures arise in astronomy, ecology, economics, biology, and sociology.

It is often the case that the objects belong to different classes that have different probability distributions. As a consequence, the sample is a mixture of such objects. The methods for studying problems of this kind appeared at the end of the nineteenth century in the papers by Newcomb [7] and Pearson [8]. The models with varying concentrations of components became popular quite recently. These models appear in various cases where the sampling is done under circumstances that are changing with time. A number of examples and a development of the relevant theory can be found in [4, 5].

We consider a certain generalization of the problem of analysis of mixtures with varying concentrations. In contrast to the classical model, the observations are dependent in our case, since the sample of objects is a result of a random sampling from a finite general population. The dependence becomes essential if the size of the general population is reasonably small.

We restrict our consideration to the case of two components. In other words, we assume that each object belongs to one of two different classes and that all objects are distributed among several groups. The total amount of objects in each of the two classes is known. The aim of the sampling is to estimate the mean values of characteristics of objects for both classes. We consider two possible approaches to describe the sampling procedure.

An important feature of the model under consideration is that the general population contains objects belonging to two different classes. It is not possible to determine exactly

2010 Mathematics Subject Classification. Primary 62G05; Secondary 62D05.

Key words and phrases. Estimation in a model of mixtures, sampling method, nonparametric estimation. 
the class to which an object belongs, while the distribution of the objects among the classes can be estimated by sampling from certain groups of objects. For example, a question about the class of an object can be a sensitive matter and usually is not asked in sociology, since for the reason of confidentiality many direct answers may be erroneous. In such a case, a more reliable procedure is to perform an anonymous questioning of groups of respondents, which allows one to avoid evasive answer bias and thus to estimate the number of members in each class. Then the sampling analysis of characteristics is conducted. The estimators studied in this paper allow one to estimate the mean values of characteristics for two classes of respondents.

An example of the situation described above is a survey analysis of how smoking affects the achievement of children in school. There are two sets of children in this case, distributed among school classes (groups). One of the approaches is to conduct an anonymous sampling survey concerning smoking in the school classes. Then one can draw conclusions about the proportions of smokers in classes. The next step is to conduct a regular sampling survey to get necessary characteristics.

The mean values can be estimated with the help of weighted sums of characteristics of the objects. The aim of such an estimation is to determine the best estimates of the mean values.

Section 2 introduces the main notation and considers two possible ways for setting up the problem. A general form of estimators and their variances is given in Section 3 . Estimators with minimax coefficients defined in [5] are considered in Section 4. Adaptive estimators are considered in Section 5. We study the asymptotic behavior of adaptive estimators and prove that they are consistent and asymptotically normal. After this, concluding remarks are given in Section 6 and proofs are demonstrated in Section 7.

\section{Setting of the PRoblem}

Assume that there are two classes of objects distributed among $K$ groups. Let the group $i$ contain $N_{i}^{1}$ objects of the first class and $N_{i}^{2}$ objects of the second class. The total amount of objects in the group $i$ is denoted by $N_{i}$. Let the total amount of objects belonging to the first and second classes be $N_{(1)}$ and $N_{(2)}$, respectively. The proportions of the objects belonging to the first and second classes in the groups are called the concentrations. The concentrations are denoted as follows:

$$
w_{i}^{l}=\frac{N_{i}^{l}}{N_{i}}, \quad i=1,2, \ldots, K, l=1,2 .
$$

Assume that the objects are listed in each group in such a way that the first $N_{i}^{1}$ objects in the list belong to the first class and they are followed by $N_{i}^{2}$ objects of the second class. A certain numerical characteristic is assigned to each object. The values of the characteristic in the group $i$ are denoted by

$$
X_{i 1}, X_{i 2}, \ldots, X_{i N_{i}}
$$

We choose $n_{i} \geq 1$ objects from the group $i$ by the simple random sampling without replacement. Denote the classes of these objects by $C_{i 1}, C_{i 2}, \ldots, C_{i n_{i}}$ and their characteristics by $Y_{i 1}, Y_{i 2}, \ldots, Y_{i n_{i}}$.

We consider two ways for sampling the objects. The first of them arises if a population is finite, and the second relies on a random choice.

In the first case, the set of all objects is fixed; however, a rearrangement of the objects into groups is possible. We assume that all those rearrangements are equally probable. We introduce the notation for the mean values and variances of the characteristics in the 
classes, namely

$$
\begin{array}{cl}
m_{1}=\frac{1}{N_{(1)}} \sum_{i=1}^{K} \sum_{j=1}^{N_{i}^{1}} X_{i j}, & m_{2}=\frac{1}{N_{(2)}} \sum_{i=1}^{K} \sum_{j=N_{i}^{1}+1}^{N_{i}} X_{i j}, \\
s_{1}^{2}=\frac{1}{N_{(1)}-1} \sum_{i=1}^{K} \sum_{j=1}^{N_{i}^{1}}\left(X_{i j}-m_{1}\right)^{2}, & s_{2}^{2}=\frac{1}{N_{(2)}-1} \sum_{i=1}^{K} \sum_{j=N_{i}^{1}+1}^{N_{i}}\left(X_{i j}-m_{2}\right)^{2} .
\end{array}
$$

The index $\mathbf{p}$ is used with the signs of the mathematical expectations, variances, and covariances to denote the averaging with respect to all possible rearrangements of objects in groups and samples. Such an averaging is called populational averaging or an averaging for a fixed population of objects. The corresponding mean values and variances are called population mean values and variances, respectively.

We consider the estimation problem for the mean values $m_{1}$ and $m_{2}$ from the sample

$$
\left\{Y_{i j}, i=1,2, \ldots, K, j=1,2, \ldots, n_{i}\right\}
$$

if $N_{i}^{l}, i=1,2, \ldots, K, l=1,2$, are known.

In the second case, we assume that the characteristics of the objects

$$
\left\{X_{i j}, i=1,2, \ldots, K, j=1,2, \ldots, N_{i}\right\}
$$

are independent random variables whose distribution is the same for all objects of the same class. These distributions are assumed to have finite second moments. The mean values and variances are denoted by

$$
\begin{array}{ll}
\mu_{1}=\mathrm{E} X_{i j}, & \sigma_{1}^{2}=\operatorname{Var} X_{i j}, \quad i=1,2, \ldots, K, j=1,2, \ldots, N_{i}^{1}, \\
\mu_{2}=\mathrm{E} X_{i j}, & \sigma_{2}^{2}=\operatorname{Var} X_{i j}, \quad i=1,2, \ldots, K, j=N_{i}^{1}+1, \ldots, N_{i} .
\end{array}
$$

In what follows the signs of the mathematical expectation, variance, and covariance correspond to the averaging with respect to characteristics $\left\{X_{i j}\right\}$ and to all possible samples from the groups. This kind of averaging is called unconditional. The corresponding mean values and variances are called the model mean values and variances.

We consider the estimation problem for the model mean values $\mu_{1}$ and $\mu_{2}$ from the sample $\left\{Y_{i j}, i=1,2, \ldots, K, j=1,2, \ldots, n_{i}\right\}$ if $N_{i}^{l}, i=1,2, \ldots, K, l=1,2$, are known.

\section{Construction of estimators}

The idea for the estimation is to use the differences between the concentrations $w_{i}^{1}$ in different groups. This idea can be achieved with the help of the estimators of the following form:

$$
\hat{m}_{l}\left(a^{l}\right)=\frac{1}{K} \sum_{i=1}^{K} a_{i}^{l} T_{i}, \quad l=1,2,
$$

where $T_{i}$ is the mean value of the characteristics in the group $i$,

$$
T_{i}=\frac{1}{n_{i}} \sum_{j=1}^{n_{i}} Y_{i j}, \quad i=1,2, \ldots, K,
$$

and where $a^{l}=\left(a_{1}^{l}, a_{2}^{l}, \ldots, a_{K}^{l}\right), l=1,2$, are some sets of coefficients.

Since the estimation procedure is the same for both classes, we consider the estimators for the first class only. In doing so, we suppress the index 1 for $a^{1}$. 
The mathematical expectation of $T_{i}$ for a fixed population is equal to

$$
\begin{aligned}
\mathrm{E}_{\mathbf{p}} T_{i} & =\frac{1}{n_{i}} \sum_{j=1}^{n_{i}} \mathrm{E}_{\mathbf{p}} Y_{i j}=\mathrm{E}_{\mathbf{p}} Y_{i 1} \\
& =\mathrm{P}_{\mathbf{p}}\left(C_{i 1}=1\right) \mathrm{E}_{\mathbf{p}}\left(Y_{i 1} \mid C_{i 1}=1\right)+\mathrm{P}_{\mathbf{p}}\left(C_{i 1}=2\right) \mathrm{E}_{\mathbf{p}}\left(Y_{i 1} \mid C_{i 1}=2\right) \\
& =w_{i}^{1} m_{1}+w_{i}^{2} m_{2}, \quad i=1,2, \ldots, K .
\end{aligned}
$$

Similarly, the mathematical expectation of the estimator $\hat{m}_{1}(a)$ for a fixed population is equal to

$$
\begin{aligned}
\mathrm{E}_{\mathbf{p}} \hat{m}_{1}(a) & =\frac{1}{K} \sum_{i=1}^{K} a_{i} \mathrm{E}_{\mathbf{p}} T_{i}=\frac{1}{K} \sum_{i=1}^{K} a_{i}\left(w_{i}^{1} m_{1}+w_{i}^{2} m_{2}\right) \\
& =\frac{m_{1}}{K} \sum_{i=1}^{K} w_{i}^{1} a_{i}+\frac{m_{2}}{K} \sum_{i=1}^{K} w_{i}^{2} a_{i} .
\end{aligned}
$$

The unconditional mathematical expectation can be written as

$$
\begin{aligned}
\mathrm{E} \hat{m}_{1}(a) & =\mathrm{EE}_{\mathbf{p}} \hat{m}_{1}(a)=\frac{\mathrm{E} m_{1}}{K} \sum_{i=1}^{K} w_{i}^{1} a_{i}+\frac{\mathrm{E} m_{2}}{K} \sum_{i=1}^{K} w_{i}^{2} a_{i} \\
& =\frac{\mu_{1}}{K} \sum_{i=1}^{K} w_{i}^{1} a_{i}+\frac{\mu_{2}}{K} \sum_{i=1}^{K} w_{i}^{2} a_{i} .
\end{aligned}
$$

In both cases, it is necessary to assume that

$$
\frac{1}{K} \sum_{i=1}^{K} w_{i}^{1} a_{i}=1, \quad \frac{1}{K} \sum_{i=1}^{K} w_{i}^{2} a_{i}=0
$$

for the unbiasedness of the estimator $\hat{m}_{1}(a)$.

In what follows we assume that these conditions hold.

Among all unbiased estimators, it is natural to choose the estimator with minimal variance. This necessarily leads to the problem of calculating the variance of the above estimator for an arbitrary set of coefficients $a_{1}, a_{2}, \ldots, a_{K}$. The following two results give the expressions for variances in both cases, for a fixed population and for randomized characteristics.

Theorem 1. The variance of the estimator $\hat{m}_{1}(a)$ in the case of a fixed population is equal to

$$
\begin{aligned}
\operatorname{Var}_{\mathbf{p}} \hat{m}_{1}(a)=\frac{1}{K^{2}} \sum_{i=1}^{K} \frac{a_{i}^{2}}{n_{i}}( & w_{i}^{1} s_{1}^{2}+w_{i}^{2} s_{2}^{2}+\frac{N_{i}-n_{i}}{N_{i}-1} w_{i}^{2} w_{i}^{1} \\
& \left.\times\left(\left(m_{1}-m_{2}\right)^{2}-\frac{s_{1}^{2}}{N_{(1)}}-\frac{s_{2}^{2}}{N_{(2)}}\right)\right)-\frac{s_{1}^{2}}{N_{(1)}} .
\end{aligned}
$$

Theorem 2. The variance of the estimator $\hat{m}_{1}(a)$ in the case of randomized characteristics is equal to

$$
\operatorname{Var} \hat{m}_{1}(a)=\frac{1}{K^{2}} \sum_{i=1}^{K} \frac{a_{i}^{2}}{n_{i}}\left(w_{i}^{1} \sigma_{1}^{2}+w_{i}^{2} \sigma_{2}^{2}+\frac{N_{i}-n_{i}}{N_{i}-1} w_{i}^{2} w_{i}^{1}\left(\mu_{1}-\mu_{2}\right)^{2}\right) .
$$




\section{Minimax coefficients}

We see from (3) and (4) that the variances depend on unknown parameters of the models in both cases. Thus the optimal coefficients $a_{1}, a_{2}, \ldots, a_{K}$ cannot be determined from a sample.

The minimax coefficients are used in 5] to estimate the distribution functions of components of the mixture of independent observations. The minimax coefficients satisfy relations (2) and minimize the following expression:

$$
\sum_{i=1}^{K} a_{i}^{2} \rightarrow \min
$$

An application of the Lagrange multipliers shows that the minimax coefficients are given by

$$
\bar{a}_{i}=\frac{\left(1-\bar{r}_{1}\right) w_{i}^{1}+\bar{r}_{2}-\bar{r}_{1}}{\bar{r}_{2}-\bar{r}_{1}^{2}}, \quad i=1,2, \ldots, K,
$$

in the nonsingular case (that is, if $\bar{r}_{2} \bar{r}_{0}-\bar{r}_{1}^{2} \neq 0$ ) where $\bar{r}_{j}, j=1,2$, are the first two moments of the concentrations $w_{i}$,

$$
\bar{r}_{j}=\frac{1}{K} \sum_{i=1}^{K}\left(w_{i}^{1}\right)^{j}, \quad j=1,2 .
$$

The singular case appears if all the $w_{i}$ 's are the same. In this case, the model is non-identifiable and the estimation is impossible.

Although the minimax coefficients do not lead to the minimal variances in (3) or (4)), they still have some nice properties. We show in the next section that the estimators with the minimax coefficients are consistent and asymptotically normal.

\section{Asymptotic Behavior of the estimators}

We study the asymptotic behavior as $K \rightarrow \infty$ of the estimators for the unconditional case. The numbers of objects in the groups $N_{i}$, the concentrations of objects for both classes $w_{i}^{l}, l=1,2$, and the sizes of samples from groups $n_{i}$ are assumed to be fixed sequences defined for all $i \geq 1$.

We study estimators of the form (1). Thus the estimator $\mu_{1}$ with the minimax coefficients (5) is given by

$$
\bar{\mu}_{1, K}=\hat{m}_{1}\left(\bar{a}_{K}\right)=\frac{1}{K} \sum_{i=1}^{K} \bar{a}_{i, K} T_{i} .
$$

The consistency and asymptotic normality of this estimator is obtained in the following result.

Theorem 3. Assume that there exist $C>0$ and $M \geq 1$ such that $\bar{r}_{2, K}-\bar{r}_{1, K}^{2}>C$ for all $K$ and $n_{i} \leq M$ for all $i \geq 1$. Then the estimator $\bar{\mu}_{1, K}$ is consistent and the distributions of the random variables

$$
\frac{1}{\sqrt{\operatorname{Var} \bar{\mu}_{1, K}}}\left(\bar{\mu}_{1, K}-\mu_{1}\right)
$$

weakly converge as $K \rightarrow \infty$ to the standard normal distribution. 
We rewrite the expression for $\operatorname{Var} \hat{m}_{1}(a)$ in order to determine the best coefficients $a_{i}$. Namely,

$$
\begin{gathered}
\operatorname{Var} \hat{m}_{1}(a)=\frac{1}{K^{2}} \sum_{i=1}^{K} a_{i}^{2} d_{i} \\
d_{i}=\frac{1}{n_{i}}\left(w_{i}^{1} \sigma_{1}^{2}+w_{i}^{2} \sigma_{2}^{2}+\frac{N_{i}-n_{i}}{N_{i}-1} w_{i}^{2} w_{i}^{1}\left(\mu_{1}-\mu_{2}\right)^{2}\right), \quad i=1,2, \ldots, K .
\end{gathered}
$$

When minimizing the expression for the variance $\operatorname{Var} \hat{m}_{1}(a)$ with respect to the coefficients $a_{1}, a_{2}, \ldots, a_{K}$ we use the necessary conditions (2) for the unbiasedness. Following the Lagrange multipliers method, we show that the best weights in the nonsingular case (that is, if $\tilde{r}_{2, K} \tilde{r}_{0, K}-\tilde{r}_{1, K}^{2} \neq 0$ ) are obtained from

$$
\tilde{a}_{i, K}=\frac{\left(\tilde{r}_{0, K}-\tilde{r}_{1, K}\right) w_{i}^{1}+\tilde{r}_{2, K}-\tilde{r}_{1, K}}{d_{i}\left(\tilde{r}_{2, K} \tilde{r}_{0, K}-\tilde{r}_{1, K}^{2}\right)}, \quad i=1,2, \ldots, K,
$$

where $\tilde{r}_{j, K}$ are weighted moments of concentrations $w_{i}$ :

$$
\tilde{r}_{j, K}=\frac{1}{K} \sum_{i=1}^{K} \frac{\left(w_{i}^{1}\right)^{j}}{d_{i}}, \quad j=0,1,2 .
$$

Thus the best unbiased estimator in the class of estimators (10) is given by

$$
\tilde{\mu}_{1, K}=\hat{m}_{1}\left(\tilde{a}_{K}\right)=\frac{1}{K} \sum_{i=1}^{K} \tilde{a}_{i, K} T_{i} .
$$

The minimal value of the variance is equal to

$$
\operatorname{Var} \tilde{\mu}_{1, K}=\frac{1}{K} \frac{\tilde{r}_{2, K}-2 \tilde{r}_{1, K}+\tilde{r}_{0, K}}{\tilde{r}_{2, K} \tilde{r}_{0, K}-\tilde{r}_{1, K}^{2}} .
$$

The following result shows that the estimator $\tilde{\mu}_{1, K}$ obtained above is asymptotically normal.

Theorem 4. If all the assumptions of Theorem 3 hold, then the estimator $\tilde{\mu}_{1, K}$ is consistent and the distributions of the random variables

$$
L_{K}:=\frac{1}{\sqrt{\operatorname{Var} \tilde{\mu}_{1, K}}}\left(\tilde{\mu}_{1, K}-\mu_{1}\right)
$$

weakly converge as $K \rightarrow \infty$ to the standard normal distribution.

Unfortunately the weights $\tilde{a}$ cannot be used in practice, since the $d_{i}$ depend on the vector of unknown parameters $\gamma=\left(\mu_{1}, \mu_{2}, \sigma_{1}, \sigma_{2}\right)$. For this reason, we first construct a pilot estimator $\bar{\gamma}_{K}=\left(\bar{\mu}_{1, K}, \bar{\mu}_{2, K}, \bar{\sigma}_{1, K}, \bar{\sigma}_{2, K}\right)$ by using the minimax weights $\bar{a}_{i}$ :

$$
\begin{gathered}
\bar{\mu}_{l, K}=\frac{1}{K} \sum_{i=1}^{K} \bar{a}_{i, K}^{l} \frac{1}{n_{i}} \sum_{j=1}^{n_{i}} Y_{i j}, \quad l=1,2, \\
\bar{\sigma}_{l, K}^{2}=\frac{1}{K} \sum_{i=1}^{K} \bar{a}_{i, K}^{l} \frac{1}{n_{i}} \sum_{j=1}^{n_{i}} Y_{i j}^{2}-\bar{\mu}_{l, K}^{2}, \quad l=1,2 .
\end{gathered}
$$

Then one can construct the estimates for the variances in groups

$$
\bar{d}_{i, K}=\frac{1}{n_{i}}\left(w_{i}^{1} \bar{\sigma}_{1, K}^{2}+w_{i}^{2} \bar{\sigma}_{2, K}^{2}+\frac{N_{i}-n_{i}}{N_{i}-1} w_{i}^{1} w_{i}^{2}\left(\bar{\mu}_{1, K}-\bar{\mu}_{2, K}\right)^{2}\right), \quad i=1,2, \ldots, K,
$$


and for weighted moments

$$
\bar{r}_{i, K}=\frac{1}{K} \sum_{i=1}^{K} \frac{\left(w_{i}^{1}\right)^{j}}{\bar{d}_{i, K}}, \quad i=1,2, \ldots, K .
$$

Now one can evaluate the adaptive weights $\hat{a}_{i, K}$ :

$$
\hat{a}_{i, K}=\frac{\left(\bar{r}_{0, K}-\bar{r}_{1, K}\right) w_{i}^{1}+\bar{r}_{2, K}-\bar{r}_{1, K}}{\bar{r}_{0, K} \bar{r}_{2, K}-\bar{r}_{1, K}^{2}}, \quad i=1,2, \ldots, K .
$$

Therefore the adaptive estimator for the mean value is given by

$$
\hat{\mu}_{1, K}=\hat{m}_{1}\left(\hat{a}_{K}\right)=\frac{1}{K} \sum_{i=1}^{K} \hat{a}_{i, K} T_{i} .
$$

We show that if the assumptions of Theorem 3 hold, then the asymptotic behavior of the adaptive estimator is the same as the asymptotic behavior of the estimator constructed from the one with best coefficients.

We consider the weight coefficients $\tilde{a}_{i, K}, i=1,2, \ldots, K$, as functions of the unknown parameters $\gamma$. Below is a particular case of Lemma 3.2.1 of [5] corresponding to $g(x)=x$ and $m=2$ :

Proposition 1. Let $\bar{r}_{2, K}-\bar{r}_{1, K}^{2}>C$ for some $C>0$ and all $K$. Then

$$
\sup _{K \in \mathbb{N}} \mathrm{P}\left(\tau_{\varepsilon}(K)>\delta\right) \rightarrow 0, \quad \varepsilon \rightarrow \infty,
$$

for all $\delta>0$, where

$$
\tau_{\varepsilon}(K)=\sup _{\alpha:|\alpha-\gamma|<\varepsilon} \frac{1}{\sqrt{\operatorname{Var} \tilde{\mu}_{1, K}}}\left|\hat{m}_{1}\left(\tilde{a}_{K}(\alpha)\right)-\hat{m}_{1}\left(\tilde{a}_{K}(\gamma)\right)\right| .
$$

Now we turn to the property of the asymptotic normality of the adaptive estimator.

Theorem 5. Assume that all the assumptions of Theorem 3 hold. Then the estimator $\hat{\mu}_{1, K}$ is consistent and the distributions of the random variables

$$
Z_{K}:=\frac{1}{\sqrt{\operatorname{Var} \tilde{\mu}_{1, K}}}\left(\hat{\mu}_{1, K}-\mu_{1}\right)
$$

weakly converge as $K \rightarrow \infty$ to the standard normal distributions.

Proof. Consider the random variables $L_{K}$ defined in Theorem 4 . For all $\delta>0$,

$$
J_{K}:=\mathrm{P}\left(\left|Z_{K}-L_{K}\right|>\delta\right) \leq \mathrm{P}\left(\tau_{\varepsilon}(K)>\delta\right)+\mathrm{P}\left(\left|\bar{\gamma}_{K}-\gamma\right|>\varepsilon\right) .
$$

Fix an arbitrary $\lambda>0$. According to Proposition 1 stated above, one can choose $\varepsilon>0$ such that

$$
\mathrm{P}\left(\tau_{\varepsilon}(K)>\delta\right)<\lambda
$$

for all $K$. It now follows from Theorem 3 that the estimators for the mean value and variance constructed from minimax coefficients (7)-(8) are consistent, whence $\bar{\gamma}_{K} \rightarrow \gamma$ in probability as $K \rightarrow \infty$. Thus, if $K$ is sufficiently large,

$$
\mathbf{P}\left(\left|\bar{\gamma}_{K}-\gamma\right|>\varepsilon\right)<\lambda \text {. }
$$

Therefore $J_{K}<2 \lambda$ for such a number $K$. Since $\lambda>0$ is arbitrary, this implies that $J_{K} \rightarrow 0$ or $\left|Z_{K}-L_{K}\right| \rightarrow 0$ in probability.

By Theorem 4, the distributions of $L_{K}$ weakly converge to the standard normal distribution. Hence the distributions of $Z_{K}$ converge to the standard normal distribution, too. 


\section{Concluding Remarks}

We generalize some techniques for studying mixtures with varying concentrations to the case of dependent observations.

The idea to use the adaptive procedure allows one to improve the asymptotic properties of the estimators. If the number $K$ is not large enough, then, when estimating $\gamma$, an extra error may cause the loss of quality of adaptive estimators. Therefore a decision to use adaptive estimators is not clear in each particular situation and requires additional research.

An extension of the technique of this paper to the case of mixtures of more than two components is suggested for further investigation.

\section{Proofs}

Proof of Theorem 1. First we evaluate the variance. We have

(9) $\operatorname{Var}_{\mathbf{p}} \hat{m}_{1}(a)=\operatorname{Var}_{\mathbf{p}} \frac{1}{K} \sum_{i=1}^{K} a_{i} T_{i}=\frac{1}{K^{2}} \sum_{i=1}^{K} a_{i}^{2} \operatorname{Var}_{\mathbf{p}} T_{i}+\frac{1}{K^{2}} \sum_{\substack{i, j=1 \\ i \neq j}}^{K} a_{i} a_{j} \operatorname{Cov}\left(T_{i}, T_{j}\right)$.

The averaging with respect to samples is denoted with the help of the index s. Then

$$
\operatorname{Var}_{\mathbf{p}} T_{i}=\mathrm{E}_{\mathbf{p}} \mathrm{E}_{\mathbf{s}}\left(T_{i}-\mathrm{E}_{\mathbf{p}} T_{i}\right)^{2}=\mathrm{E}_{\mathbf{p}} \operatorname{Var}_{\mathbf{s}} T_{i}+\mathrm{E}_{\mathbf{p}}\left(\mathrm{E}_{\mathbf{s}} T_{i}-\mathrm{E}_{\mathbf{p}} T_{i}\right)^{2} .
$$

The set of characteristics of elements of the class $l$ is denoted by $Z_{1}^{l}, Z_{2}^{l}, \ldots, Z_{N_{(l)}}^{l}$, $l=1,2$. We also put $\tilde{m}_{i}=\mathrm{E}_{\mathbf{s}} T_{i}$ and $\bar{m}_{i}=\mathrm{E}_{\mathbf{p}} T_{i}, i=1,2, \ldots, K$. It is easy to see that

$$
\tilde{m}_{i}=\frac{1}{N_{i}} \sum_{j=1}^{N_{i}} X_{i j}, \quad \operatorname{Var}_{\mathbf{s}} T_{i}=\frac{N_{i}-n_{i}}{N_{i}\left(N_{i}-1\right) n_{i}} \sum_{j=1}^{N_{i}}\left(X_{i j}-\tilde{m}_{i}\right)^{2}, \quad i=1,2, \ldots, K .
$$

In what follows we need the following auxiliary result.

Lemma 1. Let $\left\{Z_{1}, Z_{2}\right\}$ be a simple random sample without replacement from a population of characteristics of objects $Z_{1}^{l}, Z_{2}^{l}, \ldots, Z_{N_{(l)}}^{l}, l=1,2$, of the class $l$. Then

1. $\operatorname{Cov}\left(Z_{1}, Z_{2}\right)=-s_{l}^{2} / N_{(l)}$.

2. $\operatorname{Cov}\left(Y_{i 1}, Y_{j 1}\right)=-w_{i}^{1} w_{j}^{1} s_{1}^{2} / N_{(1)}-w_{i}^{2} w_{j}^{2} s_{2}^{2} / N_{(2)}$.

3. $\mathrm{E}_{\mathbf{p}}\left(\tilde{m}_{i}-\bar{m}_{i}\right)^{2}=\frac{1}{N_{i}}\left(w_{i}^{1} s_{1}^{2}+w_{i}^{2} s_{2}^{2}\right)-\left(w_{i}^{1}\right)^{2} s_{1}^{2} / N_{(1)}-\left(w_{i}^{2}\right)^{2} s_{2}^{2} / N_{(2)}$.

Proof. The lemma follows from the following expressions for the covariances:

$$
\begin{aligned}
\operatorname{Cov}\left(Z_{1}, Z_{2}\right) & =Z_{1} Z_{2}-\mathrm{E}_{\mathbf{p}} Z_{1} \mathrm{E}_{\mathbf{p}} Z_{2} \\
& =\frac{1}{N_{(l)}\left(N_{(l)}-1\right)} \sum_{i, j=1, i \neq j}^{N_{(l)}} Z_{i}^{l} Z_{j}^{l}-m_{l}^{2} \\
& =N_{(l)}\left(N_{(l)}-1\right)\left(\left(N_{(l)}\right)^{2} m_{l}^{2}-\sum_{i=1}^{N_{(l)}}\left(Z_{i}^{l}\right)^{2}\right)-m_{l}^{2} \\
& =N_{(l)}\left(N_{(l)}-1\right)\left(\left(N_{(l)}\right)^{2} m_{l}^{2}-\left(N_{(l)}-1\right) s_{1}^{2}-N_{(l)} m_{l}^{2}\right)-m_{l}^{2} \\
& =-\frac{s_{l}^{2}}{N_{(l)}}
\end{aligned}
$$


and

$$
\begin{aligned}
\operatorname{Cov}\left(Y_{i 1}, Y_{j 1}\right) & =\operatorname{Cov}\left(Y_{i 1} \mathbf{1}_{\left\{C_{i 1}=1\right\}}+Y_{i 1} \mathbf{1}_{\left\{C_{i 1}=2\right\}}, Y_{j 1} \mathbf{1}_{\left\{C_{j 1}=1\right\}}+Y_{j 1} \mathbf{1}_{\left\{C_{j 1}=2\right\}}\right) \\
& =\operatorname{Cov}\left(Y_{i 1} \mathbf{1}_{\left\{C_{i 1}=1\right\}}, Y_{j 1} \mathbf{1}_{\left\{C_{j 1}=1\right\}}\right)+\operatorname{Cov}\left(Y_{i 1} \mathbf{1}_{\left\{C_{i 1}=2\right\}}, Y_{j 1} \mathbf{1}_{\left\{C_{j 1}=2\right\}}\right) \\
& =-w_{i}^{1} w_{j}^{1} \frac{s_{1}^{2}}{N_{(1)}}-w_{i}^{2} w_{j}^{2} \frac{s_{2}^{2}}{N_{(2)}}
\end{aligned}
$$

and those for the variances

$$
\begin{aligned}
\mathrm{E}_{\mathbf{p}}\left(\tilde{m}_{i}-\bar{m}_{i}\right)^{2}= & \mathrm{E}_{\mathbf{p}}\left(\frac{1}{N_{i}} \sum_{j=1}^{N_{i}} X_{i j}-\bar{m}_{i}\right)^{2} \\
= & \mathrm{E}_{\mathbf{p}}\left(w_{i}^{1}\left(\sum_{j=1}^{N_{i}^{1}} X_{i j}-m_{1}\right)+w_{i}^{2}\left(\sum_{j=N_{i}^{1}+1}^{N_{i}} X_{i j}-m_{2}\right)\right)^{2} \\
= & \left(w_{i}^{1}\right)^{2} \mathrm{E}_{\mathbf{p}}\left(\sum_{j=1}^{N_{i}^{1}} X_{i j}-m_{1}\right)^{2} \\
& +\left(w_{i}^{2}\right)^{2} \mathrm{E}_{\mathbf{p}}\left(\sum_{j=N_{i}^{1}+1}^{N_{i}} X_{i j}-m_{2}\right)^{2} \\
= & \left(w_{i}^{1}\right)^{2}\left(1-\frac{N_{i}^{1}}{N_{(1)}}\right) \frac{s_{1}^{2}}{N_{i}^{1}}+\left(w_{i}^{2}\right)^{2}\left(1-\frac{N_{i}^{2}}{N_{(2)}}\right) \frac{s_{2}^{2}}{N_{i}^{2}} \\
= & w_{i}^{1} s_{1}^{2}+w_{i}^{2} s_{2}^{2}-\left(w_{i}^{1}\right)^{2} \frac{s_{1}^{2}}{N_{(1)}}-\left(w_{i}^{2}\right)^{2} \frac{s_{2}^{2}}{N_{(2)}}
\end{aligned}
$$

Thus the variances are given by

$$
\begin{aligned}
\operatorname{Var}_{\mathbf{p}} T_{i}= & \frac{N_{i}-n_{i}}{N_{i}\left(N_{i}-1\right) n_{i}} \sum_{j=1}^{N_{i}} \mathrm{E}_{\mathbf{p}}\left(X_{i j}-\tilde{m}_{i}\right)^{2}+\mathrm{E}_{\mathbf{p}}\left(\bar{m}_{i}-\tilde{m}_{i}\right)^{2} \\
= & \frac{N_{i}-n_{i}}{N_{i}\left(N_{i}-1\right) n_{i}} \sum_{j=1}^{N_{i}}\left(\mathrm{E}_{\mathbf{p}}\left(X_{i j}-\bar{m}_{i}\right)^{2}-\sum_{j=1}^{N_{i}} \mathrm{E}_{\mathbf{p}}\left(\bar{m}_{i}-\tilde{m}_{i}\right)^{2}\right)+\mathrm{E}_{\mathbf{p}}\left(\bar{m}_{i}-\tilde{m}_{i}\right)^{2} \\
= & \frac{N_{i}-n_{i}}{N_{i}\left(N_{i}-1\right) n_{i}} \sum_{j=1}^{N_{i}} \mathrm{E}_{\mathbf{p}}\left(X_{i j}-\bar{m}_{i}\right)^{2}+\frac{N_{i}\left(n_{i}-1\right)}{\left(N_{i}-1\right) n_{i}} \mathrm{E}_{\mathbf{p}}\left(\bar{m}_{i}-\tilde{m}_{i}\right)^{2} \\
= & \frac{N_{i}-n_{i}}{\left(N_{i}-1\right) n_{i}}\left(w_{i}^{1} \mathrm{E}_{\mathbf{p}}\left(\left(Y_{i 1}-\bar{m}_{i}\right)^{2} \mid C_{i 1}=1\right)+w_{i}^{2} \mathrm{E}_{\mathbf{p}}\left(\left(Y_{i 1}-\bar{m}_{i}\right)^{2} \mid C_{i 1}=2\right)\right) \\
& +\frac{N_{i}\left(n_{i}-1\right)}{\left(N_{i}-1\right) n_{i}} \mathrm{E}_{\mathbf{p}}\left(\bar{m}_{i}-\tilde{m}_{i}\right)^{2} \\
= & \frac{N_{i}-n_{i}}{\left(N_{i}-1\right) n_{i}}\left[w_{i}^{1}\left(\mathrm{E}_{\mathbf{p}}\left(\left(Y_{i 1}-m_{1}\right)^{2} \mid C_{i 1}=1\right)+\mathrm{E}_{\mathbf{p}}\left(\bar{m}_{i}-m_{1}\right)^{2}\right)\right. \\
& \left.\quad+w_{i}^{2}\left(\mathrm{E}_{\mathbf{p}}\left(\left(Y_{i 1}-m_{2}\right)^{2} \mid C_{i 1}=2\right)+\mathrm{E}_{\mathbf{p}}\left(\bar{m}_{i}-m_{2}\right)\right)\right] \\
& +\frac{N_{i}\left(n_{i}-1\right)}{\left(N_{i}-1\right) n_{i}} \mathrm{E}_{\mathbf{p}}\left(\bar{m}_{i}-\tilde{m}_{i}\right)^{2}
\end{aligned}
$$




$$
\begin{aligned}
= & \frac{N_{i}-n_{i}}{\left(N_{i}-1\right) n_{i}}\left(w_{i}^{1} \frac{N_{(1)}-1}{N_{(1)}} s_{1}^{2}+w_{i}^{2} \frac{N_{(2)}-1}{N_{(2)}} s_{2}^{2}\right. \\
& \left.\quad+w_{i}^{1}\left(w_{i}^{2}\right)^{2}\left(m_{1}-m_{2}\right)^{2}+w_{i}^{2}\left(w_{i}^{1}\right)^{2}\left(m_{1}-m_{2}\right)^{2}\right) \\
& +\frac{n_{i}-1}{\left(N_{i}-1\right) n_{i}}\left(w_{i}^{1} s_{1}^{2}+w_{i}^{2} s_{2}^{2}\right)-\frac{N_{i}\left(n_{i}-1\right)}{\left(N_{i}-1\right) n_{i}}\left(\left(w_{i}^{1}\right)^{2} \frac{s_{1}^{2}}{N_{(1)}}+\left(w_{i}^{2}\right)^{2} \frac{s_{2}^{2}}{N_{(2)}}\right) \\
= & \frac{1}{n_{i}}\left(w_{i}^{1} s_{1}^{2}+w_{i}^{2} s_{2}^{2}+\frac{N_{i}-n_{i}}{N_{i}-1}\left(w_{i}^{2} w_{i}^{1}\left(m_{1}-m_{2}\right)^{2}-w_{i}^{1} \frac{s_{1}^{2}}{N_{(1)}}-w_{i}^{2} \frac{s_{2}^{2}}{N_{(2)}}\right)\right) \\
& -\frac{N_{i}\left(n_{i}-1\right)}{\left(N_{i}-1\right) n_{i}}\left(\left(w_{i}^{1}\right)^{2} \frac{s_{1}^{2}}{N_{(1)}}+\left(w_{i}^{2}\right)^{2} \frac{s_{2}^{2}}{N_{(2)}}\right) .
\end{aligned}
$$

Further we transform the sum with the covariances in (9),

$$
\begin{aligned}
\frac{1}{K^{2}} \sum_{\substack{i, j=1 \\
i \neq j}}^{K} a_{i} a_{j} \operatorname{Cov}\left(T_{i}, T_{j}\right) & =\frac{1}{K^{2}} \sum_{\substack{i, j=1 \\
i \neq j}}^{K} a_{i} a_{j} \operatorname{Cov}\left(\frac{1}{n_{i}} \sum_{j=1}^{n_{i}} Y_{i k}, \frac{1}{n_{i}} \sum_{j=1}^{n_{i}} Y_{j k}\right) \\
& =\frac{1}{K^{2}} \sum_{\substack{i, j=1 \\
i \neq j}}^{K} a_{i} a_{j} \operatorname{Cov}\left(Y_{i 1}, Y_{j 1}\right) \\
& =-\frac{1}{K^{2}} \sum_{\substack{i, j=1 \\
i \neq j}}^{K} a_{i} a_{j}\left(w_{i}^{1} w_{j}^{1} \frac{s_{1}^{2}}{N_{(1)}}-w_{i}^{2} w_{j}^{2} \frac{s_{2}^{2}}{N_{(2)}}\right) \\
& =-\frac{s_{1}^{2}}{N_{(1)}}+\frac{1}{K^{2}} \sum_{i=1}^{K} a_{i}^{2}\left(\left(w_{i}^{1}\right)^{2} \frac{s_{1}^{2}}{N_{(1)}}-\left(w_{i}^{2}\right)^{2} \frac{s_{2}^{2}}{N_{(2)}}\right) .
\end{aligned}
$$

As a result, expression (9) is rewritten as follows:

$$
\begin{aligned}
& \frac{1}{K^{2}} \sum_{i=1}^{K} a_{i}^{2} \operatorname{Var}_{\mathbf{p}} T_{i}+\frac{1}{K^{2}} \sum_{\substack{i, j=1 \\
i \neq j}}^{K} a_{i} a_{j} \operatorname{Cov}\left(T_{i}, T_{j}\right) \\
&=\frac{1}{K^{2}} \sum_{i=1}^{K} a_{i}^{2}\left[\frac { 1 } { n _ { i } } \left(w_{i}^{1} s_{1}^{2}+w_{i}^{2} s_{2}^{2}\right.\right. \\
&\left.\quad+\frac{N_{i}-n_{i}}{N_{i}-1}\left(w_{i}^{2} w_{i}^{1}\left(m_{1}-m_{2}\right)^{2}-w_{i}^{1} \frac{s_{1}^{2}}{N_{(1)}}-w_{i}^{2} \frac{s_{2}^{2}}{N_{(2)}}\right)\right) \\
&\left.\quad-\frac{N_{i}\left(n_{i}-1\right)}{\left(N_{i}-1\right) n_{i}}\left(\left(w_{i}^{1}\right)^{2} \frac{s_{1}^{2}}{N_{(1)}}+\left(w_{i}^{2}\right)^{2} \frac{s_{2}^{2}}{N_{(2)}}\right)\right] \\
&+\frac{1}{K^{2}} \sum_{i=1}^{K} a_{i}^{2}\left(\left(w_{i}^{1}\right)^{2} \frac{s_{1}^{2}}{N_{(1)}}-\left(w_{i}^{2}\right)^{2} \frac{s_{2}^{2}}{N_{(2)}}\right)-\frac{s_{1}^{2}}{N_{(1)}} \\
&=\frac{1}{K^{2}} \sum_{i=1}^{K} a_{i}^{2}\left[\frac { 1 } { n _ { i } } \left(w_{i}^{1} s_{1}^{2}+w_{i}^{2} s_{2}^{2}\right.\right. \\
&\left.\quad+\frac{N_{i}-n_{i}}{N_{i}-1}\left(w_{i}^{2} w_{i}^{1}\left(m_{1}-m_{2}\right)^{2}-w_{i}^{1} \frac{s_{1}^{2}}{N_{(1)}}-w_{i}^{2} \frac{s_{2}^{2}}{N_{(2)}}\right)\right) \\
&\left.\quad+\frac{N_{i}-n_{i}}{\left(N_{i}-1\right) n_{i}}\left(\left(w_{i}^{1}\right)^{2} \frac{s_{1}^{2}}{N_{(1)}}+\left(w_{i}^{2}\right)^{2} \frac{s_{2}^{2}}{N_{(2)}}\right)\right]-\frac{s_{1}^{2}}{N_{(1)}}
\end{aligned}
$$




$$
\begin{aligned}
= & \frac{1}{K^{2}} \sum_{i=1}^{K} \frac{a_{i}^{2}}{n_{i}}\left(w_{i}^{1} s_{1}^{2}+w_{i}^{2} s_{2}^{2}+\frac{N_{i}-n_{i}}{N_{i}-1} w_{i}^{2} w_{i}^{1}\left(\left(m_{1}-m_{2}\right)^{2}-\frac{s_{1}^{2}}{N_{(1)}}-\frac{s_{2}^{2}}{N_{(2)}}\right)\right) \\
& -\frac{s_{1}^{2}}{N_{(1)}} .
\end{aligned}
$$

Proof of Theorem 2. The unconditional mathematical expectation can be evaluated via the conditional one for a fixed population, namely

$$
\operatorname{Var} \hat{m}_{1}(a)=\mathrm{E}\left(\hat{m}_{1}(a)-m_{1}\right)^{2}+\mathrm{E}\left(m_{1}-\mu_{1}\right)^{2}=\operatorname{EVar}_{\mathbf{p}} \hat{m}_{1}(a)+\operatorname{Var} m_{1} .
$$

The definitions of the population means and variances imply that

$$
\mathrm{E} m_{l}=\mu_{l}, \quad \operatorname{Var} m_{l}=\frac{\sigma_{1}^{2}}{N_{(1)}}, \quad \mathrm{E} s_{l}^{2}=\sigma_{l}^{2}, \quad l=1,2 .
$$

Next we evaluate the unconditional mathematical expectation of the square of the difference between population means:

$$
\begin{aligned}
\mathrm{E}\left(m_{1}-m_{2}\right)^{2} & =\mathrm{E}\left(\frac{1}{N_{(1)}} \sum_{i=1}^{N_{(1)}} X_{i}^{1}-\frac{1}{N_{(2)}} \sum_{i=1}^{N_{(2)}} X_{i}^{2}\right)^{2} \\
& =\left(\mu_{1}-\mu_{2}\right)^{2}+\mathrm{E}\left(\frac{1}{N_{(1)}} \sum_{i=1}^{N_{(1)}} X_{i}^{1}-\mu_{1}\right)^{2}+\mathrm{E}\left(\frac{1}{N_{(2)}} \sum_{i=1}^{N_{(2)}} X_{i}^{2}-\mu_{2}\right)^{2} \\
& =\left(\mu_{1}-\mu_{2}\right)^{2}+\operatorname{Var} \frac{1}{N_{(1)}} \sum_{i=1}^{N_{(1)}} X_{i}^{1}+\operatorname{Var} \frac{1}{N_{(2)}} \sum_{i=1}^{N_{(2)}} X_{i}^{2} \\
& =\left(\mu_{1}-\mu_{2}\right)^{2}+\frac{\sigma_{1}^{2}}{N_{(1)}}+\frac{\sigma_{2}^{2}}{N_{(2)}}
\end{aligned}
$$

Then we obtain the unconditional variance of the estimator $\hat{m}_{1}(a)$ :

$$
\begin{gathered}
\operatorname{Var} \hat{m}_{1}(a)=\mathrm{E}\left[\frac { 1 } { K ^ { 2 } } \sum _ { i = 1 } ^ { K } \frac { a _ { i } ^ { 2 } } { n _ { i } } \left(w_{i}^{1} s_{1}^{2}+w_{i}^{2} s_{2}^{2}+\frac{N_{i}-n_{i}}{N_{i}-1} w_{i}^{2} w_{i}^{1}\right.\right. \\
\left.\left.\quad \times\left(\left(m_{1}-m_{2}\right)^{2}-\frac{s_{1}^{2}}{N_{(1)}}-\frac{s_{2}^{2}}{N_{(2)}}\right)\right)-\frac{s_{1}^{2}}{N_{(1)}}\right]+\frac{\sigma_{1}^{2}}{N_{(1)}} \\
=\frac{1}{K^{2}} \sum_{i=1}^{K} \frac{a_{i}^{2}}{n_{i}}\left(w_{i}^{1} \sigma_{1}^{2}+w_{i}^{2} \sigma_{2}^{2}+\frac{N_{i}-n_{i}}{N_{i}-1} w_{i}^{2} w_{i}^{1}\left(\mu_{1}-\mu_{2}\right)^{2}\right) .
\end{gathered}
$$

Proof of Theorem 3. Note that the assumptions of the theorem yield the boundedness of the coefficients $a_{i, K}$, that is,

$$
\begin{aligned}
\left|\bar{a}_{i, K}\right| & =\left|\frac{\left(1-\bar{r}_{1, K}\right) w_{i}^{1}+\bar{r}_{2, K}-\bar{r}_{1, K}}{\bar{r}_{2, K}-\bar{r}_{1, K}^{2}}\right| \leq \frac{1-\bar{r}_{1, K}+\left|\bar{r}_{2, K}-\bar{r}_{1, K}\right|}{\bar{r}_{2, K}-\bar{r}_{1, K}^{2}} \\
& =\frac{1-\bar{r}_{2, K}}{\bar{r}_{2, K}-\bar{r}_{1, K}^{2}} \leq \frac{1}{C}, \quad i=1,2, \ldots, K .
\end{aligned}
$$

The following central limit theorem of [2] is needed to prove the asymptotic normality of the estimators with minimax coefficients.

Proposition 2 (Lindeberg's central limit theorem for standard series). Let a sequence $\zeta_{n i}$ satisfy the following conditions. 
(1) Independence: the random variables $\left\{\zeta_{n 1}, \zeta_{n 2}, \ldots, \zeta_{n n}\right\}$ are jointly independent in every series.

(2) Centering: $\mathrm{E} \zeta_{n i}=0$ for $n \geq 1$ and $i=1,2, \ldots, n$.

(3) Normalization: the variances $\operatorname{Var} \zeta_{n i}=\sigma_{n i}^{2}$ exist and

$$
\sum_{i=1}^{n} \operatorname{Var} \zeta_{n i}=1 \text {. }
$$

(4) Lindeberg condition: for all $\varepsilon>0$,

$$
L_{n}(\varepsilon):=\sum_{i=1}^{n} \mathrm{E} \zeta_{n i}^{2} \mathbf{1}_{\left\{\left|\zeta_{n i}\right|>\varepsilon\right\}} \rightarrow 0
$$

as $n \rightarrow \infty$.

Then the distributions of the sums $S_{n}=\sum_{i=1}^{n} \zeta_{n i}$ weakly converge as $n \rightarrow \infty$ to the standard normal distribution.

Put

$$
\xi_{i, K}=\frac{\bar{a}_{i, K}}{K \sqrt{\operatorname{Var} \bar{\mu}_{1, K}}}\left(T_{i}-\mathrm{E} T_{i}\right) .
$$

Then we check the assumptions of Lindeberg's central limit theorem for the standard series of the random variables

$$
S_{K}=\frac{\bar{\mu}_{1, K}-\mu_{1}}{\sqrt{\operatorname{Var} \bar{\mu}_{1, K}}}=\sum_{i=1}^{K} \xi_{i, K} .
$$

Condition (1) of Proposition 2 holds, since the sampling from groups is performed randomly according to the setting of the problem. Conditions (2)-(3) of Proposition 2 follow from the definition of $\xi_{i, K}$.

Now we estimate $L_{n}(\varepsilon)$. The variance of the estimator $\hat{m}_{1}(\bar{a})$ is estimated as follows:

$$
\begin{aligned}
\operatorname{Var} \bar{\mu}_{1, K} & =\frac{1}{K^{2}} \sum_{i=1}^{K} \frac{\bar{a}_{i, K}^{2}}{n_{i}}\left(w_{i}^{1} \sigma_{1}^{2}+w_{i}^{2} \sigma_{2}^{2}+\frac{N_{i}-n_{i}}{N_{i}-1} w_{i}^{2} w_{i}^{1}\left(\mu_{1}-\mu_{2}\right)^{2}\right) \\
& \geq \frac{\min \left(\sigma_{1}^{2}, \sigma_{2}^{2}\right)}{M K^{2}} \sum_{i=1}^{K} \bar{a}_{i, K}^{2} .
\end{aligned}
$$

Next, we use the expression for the coefficients $\bar{a}_{i, K}$ and obtain from equalities (2) that

$$
\begin{aligned}
\frac{1}{K} \sum_{i=1}^{K} \bar{a}_{i, K}^{2} & =\frac{1}{K} \sum_{i=1}^{K} \frac{\left(1-\bar{r}_{1, K}\right) w_{i}^{1} a_{i, K}+\left(\bar{r}_{2, K}-\bar{r}_{1, K}\right) a_{i, K}}{\bar{r}_{2, K}-\bar{r}_{1, K}^{2}}=\frac{\bar{r}_{2, K}-2 \bar{r}_{1, K}+1}{\bar{r}_{2, K}-\bar{r}_{1, K}^{2}} \\
& =1+\frac{\left(\bar{r}_{1, K}-1\right)^{2}}{\bar{r}_{2, K}-\bar{r}_{1, K}^{2}} \geq 1 .
\end{aligned}
$$

Thus

$$
\operatorname{Var} \hat{\mu}_{1, K} \geq \frac{C_{1}}{K}
$$

for some constant $C_{1}>0$, whence

$$
\begin{aligned}
\mathrm{E} \xi_{i, K}^{2} \mathbf{1}_{\left\{\left|\xi_{i, K}\right|>\tau\right\}} & =\mathrm{E} \frac{\bar{a}_{i, K}^{2}\left(T_{i}-\mathrm{E} T_{i}\right)^{2}}{K^{2} \operatorname{Var} \bar{\mu}_{1, K}} \mathbf{1}_{\left\{\left|T_{i}-\mathrm{E} T_{i}\right|>\frac{\tau K \sqrt{\operatorname{Var} \overline{\mu_{1, K}}}}{\left|\bar{a}_{i, K}\right|}\right\}} \\
& \leq \frac{1}{C^{2} C_{1} K} \mathrm{E}\left(T_{i}-\mathrm{E} T_{i}\right)^{2} \mathbf{1}_{\left\{\left|T_{i}-\mathrm{E} T_{i}\right|>C \sqrt{C_{1} K \varepsilon}\right\}} .
\end{aligned}
$$


Let $Z_{1}^{1}, Z_{2}^{1}, \ldots, Z_{M}^{1}$ and $Z_{1}^{2}, Z_{2}^{2}, \ldots, Z_{M}^{2}$ be independent random variables whose distributions are the same as those of the characteristics of objects of the first and second classes, respectively. Consider the sum of the absolute values of centered random variables,

$$
\eta=\sum_{j=1}^{M}\left|Z_{j}^{1}-\mu_{1}\right|+\sum_{j=1}^{M}\left|Z_{j}^{2}-\mu_{2}\right|
$$

Since $T_{i}$ is the mean value of at most $M$ characteristics of objects of the first and second classes,

$$
\mathrm{E}\left(T_{i}-\mathrm{E} T_{i}\right)^{2} \mathbf{1}_{\left\{\left|T_{i}-\mathrm{E} T_{i}\right|>C \sqrt{C_{1} K \varepsilon}\right\}} \leq \mathrm{E} \eta^{2} \mathbf{1}_{\left\{\eta>C \sqrt{C_{1} K \varepsilon}\right\}} .
$$

This implies that Lindeberg's condition holds,

$$
L_{n}(\varepsilon) \leq \frac{1}{C^{2} C_{1}} \mathrm{E} \eta^{2} \mathbf{1}_{\left\{\eta>C \sqrt{C_{1} K} \varepsilon\right\}} \rightarrow 0, \quad K \rightarrow \infty
$$

since the random variable $\eta$ has finite second moment.

The variance of the estimator $\bar{\mu}_{1, K}$ approaches zero:

$$
\operatorname{Var} \bar{\mu}_{1, K} \leq \frac{\sigma_{1}^{2}+\sigma_{2}^{2}+\left(\mu_{1}-\mu_{2}\right)^{2}}{K^{2}} \sum_{i=1}^{K} \bar{a}_{i, K}^{2} \leq \frac{\sigma_{1}^{2}+\sigma_{2}^{2}+\left(\mu_{1}-\mu_{2}\right)^{2}}{C^{2} K} \rightarrow 0, \quad K \rightarrow \infty .
$$

Thus the consistency of the estimator $\bar{\mu}_{1, K}$ follows from its asymptotic normality.

Proof of Theorem 4. Considering $d_{i}$, we prove that there exist constants $0<C_{1}, C_{2}<\infty$ such that $C_{1}<d_{i}<C_{2}$ for all $i$ and $K$. We rewrite the condition imposed on the coefficients $\bar{r}_{1, K}$ and $\bar{r}_{2, K}$ as follows:

$$
\begin{aligned}
C & <\bar{r}_{2, K}-\bar{r}_{1, K}^{2}=\frac{1}{K} \sum_{i=1}^{K}\left(w_{i}-\bar{r}_{1, K}\right)^{2}=\frac{1}{K} \sum_{\left|w_{i}\right| \geq \varepsilon}\left(w_{i}-\bar{r}_{1, K}\right)^{2}+\frac{1}{K} \sum_{\left|w_{i}\right|<\varepsilon}\left(w_{i}-\bar{r}_{1, K}\right)^{2} \\
& \leq \frac{1}{K} \sum_{\left|w_{i}\right| \geq \varepsilon} 1+\frac{1}{K} \sum_{\left|w_{i}\right|<\varepsilon} \varepsilon^{2} \leq \frac{h_{1}}{K}+\varepsilon^{2},
\end{aligned}
$$

where $h_{1}$ is the number of groups with concentrations $w_{i}$ for which the distance from the mean value is at least $\varepsilon$. Thus

$$
h_{1}>K\left(C-\varepsilon^{2}\right) .
$$

It is clear that at least half of such concentrations are either smaller or greater than the mean value. For definiteness, assume that $h_{2}$ concentrations are greater than $\bar{r}_{1, K}+\varepsilon$ and that $h_{2}>h_{1} / 2=K\left(C-\varepsilon^{2}\right) / 2$. Then we estimate the number of groups $h_{3}$ with concentrations that do not exceed $\bar{r}_{1, K}$ :

$$
0=\sum_{i=1}^{K}\left(w_{i}-\bar{r}_{1, K}\right)=\sum_{w_{i} \leq \bar{r}_{1, K}}\left(w_{i}-\bar{r}_{1, K}\right)+\sum_{w_{i}>\bar{r}_{1, K}}\left(w_{i}-\bar{r}_{1, K}\right) \geq-h_{3}+h_{2} \varepsilon .
$$

Thus $h_{3} \geq h_{2} \varepsilon=K \varepsilon\left(C-\varepsilon^{2}\right) / 2$ and

$$
\begin{aligned}
\tilde{r}_{2, K} \tilde{r}_{0, K}-\tilde{r}_{1, K}^{2} & =\tilde{r}_{0, K} \frac{1}{K} \sum_{i=1}^{K} \frac{\left(w_{i}-\tilde{r}_{1, K} / \tilde{r}_{0, K}\right)^{2}}{d_{i}} \geq \frac{1}{K C_{2}^{2}} \sum_{i=1}^{K}\left(w_{i}-\tilde{r}_{1, K} / \tilde{r}_{0, K}\right)^{2} \\
& \geq \frac{h_{3}}{4 K C_{2}^{2}} \varepsilon^{2}=\frac{\varepsilon^{3}\left(C-\varepsilon^{2}\right)}{8 C_{2}^{2}} .
\end{aligned}
$$

Choose $\varepsilon=C / 2$. Then

$$
\tilde{r}_{2, K} \tilde{r}_{0, K}-\tilde{r}_{1, K}^{2} \geq \frac{C^{5}}{256 C_{2}^{2}}
$$


This implies that the best weight coefficients are bounded:

$$
\left|\tilde{a}_{i, K}\right|=\frac{\left|\left(\tilde{r}_{0, K}-\tilde{r}_{1, K}\right) w_{i}^{1}+\tilde{r}_{2, K}-\tilde{r}_{1, K}\right|}{d_{i}\left(\tilde{r}_{2, K} \tilde{r}_{0, K}-\tilde{r}_{1, K}^{2}\right)} \leq \frac{\tilde{r}_{0, K}}{d_{i}\left(\tilde{r}_{2, K} \tilde{r}_{0, K}-\tilde{r}_{1, K}^{2}\right)} \leq \frac{256 C_{2}^{2}}{C^{5} C_{1}^{2}} .
$$

Put

$$
\xi_{i, K}=\frac{\tilde{a}_{i, K}}{K \sqrt{\operatorname{Var} \tilde{\mu}_{1, K}}}\left(T_{i}-\mathrm{E} T_{i}\right) .
$$

Then $L_{K}=\sum_{i=1}^{K} \xi_{i}$. Now the result of the theorem follows from the Lindeberg central limit theorem for $\xi_{i}$. The conditions of the Lindeberg central limit theorem can be checked as in the proof of Theorem 3 . Since the variances of the estimators $\operatorname{Var} \tilde{\mu}_{1, K}$ approach zero as $K \rightarrow \infty$, the consistency follows.

\section{BIBLIOGRAPHY}

1. A. A. Borovkov, Mathematical Statistics, Nauka, Moscow, 1977; English transl., Gordon and Breach Science Publishers, Amsterdam, 1998. MR782295 (86i:62001)

2. M. V. Kartashov, Probability, Processes, Statistics, Kyiv University, Kyiv, 2008. (Ukrainian)

3. R. E. Maıboroda, Estimates for distributions of components of mixtures with varying concentrations, Ukr. Matem. Zh. 48 (1996), no. 4, 562-566; English transl. in Ukrainian Math. J. 48 (1996), 618-622. MR1417019 (97j:62055)

4. R. E. Maı̌boroda, Statistical analysis of mixtures, Kyiv University, Kyiv, 2003. (Ukrainian)

5. R. E. Maı̌boroda and O. V. Sugakova, Estimation and classification after observations in a mixture, Kyiv University, Kyiv, 2008. (Ukrainian)

6. O. O. Kubaychuk, Estimation of moments by observations from mixtures with varying concentrations, Theory Stoch. Process. 8(24) (2002), no. 3-4, 226-232. MR2027394 (2005g:62065)

7. S. Newcomb, A generalized theory of the combination of observations so as to obtain the best result, Amer. J. Math. 8 (1894), 343-366. MR.1505430

8. K. Pearson, Contribution to the mathematical theory of evolution, Trans. Roy. Soc. A 185 (1894), 71-110.

9. D. M. Titterington, A. F. Smith, and U. E. Makov, Analysis of Finite Mixture Distributions, Wiley, New York, 1985. MR0838090(87j:62033)

Department of Probability Theory, Statistics, and Actuarial Mathematics, Faculty for Mechanics and Mathematics, National Taras Shevchenko University, Academician Glushkov Avenue 2, Kiev 03127, Ukraine

E-mail address: artshcherbina@gmail.com

Received 8/JUN/2010

Translated by S. V. KVASKO 